\title{
A Homicidal Differential Drive Robot
}

\author{
Ubaldo Ruiz and Rafael Murrieta-Cid \\ Centro de Investigación en Matemáticas, CIMAT \\ Guanajuato, México \\ \{ubaldo,murrieta\}@cimat.mx
}

\begin{abstract}
In this paper, we consider the problem of capturing an omnidirectional evader using a Differential Drive Robot in an obstacle free environment. At the beginning of the game the evader is at a distance $L>l$ from the pursuer. The pursuer goal is to get closer from the evader than the capture distance $l$. The goal of the evader is to keep the pursuer at all time farther from it than this capture distance. In this paper, we found closed-form representations of the motion primitives and time-optimal strategies for each player. These strategies are in Nash Equilibrium, meaning that any unilateral deviation of each player from these strategies does not provide to such player benefit toward the goal of winning the game. We also present the condition defining the winner of the game and we construct a solution over the entire reduced space.
\end{abstract}

\section{INTRODUCTION}

This paper addresses a pursuit-evasion game [9], [4], [6], [8], [7], [11]. In our past research [10], we have presented a solution for the problem of tracking an omnidirectional mobile evader at constant distance with a differential drive robot. In that work we have obtained optimal motion strategies, in the sense that they require the minimal speed for both players for winning, and the long term solution for the game, the main drawback of that work [10] is that the proposed motion strategies are not time-optimal. The distinguishing feature of our current work compared with our previous research is that the proposed motion strategies for both players are time-optimal.

The problem that we address in this work is close related to the classical differential game, called the Homicidal Chauffeur problem [3], [12]. In that game a faster pursuer (w.r.t. the evader) has as its objective to get closer than a given distance (the capture condition) from a slower but more agile evader. The pursuer is a vehicle with a minimal turning radius. In our problem, we also consider a faster pursuer and a more agile evader moving in an environment without obstacles. However, there is an important difference between both problems. In this work the pursuer is a Differential Drive Robot (DDR), i.e. the pursuer can rotate in place. The change in the mechanical model of the pursuer has as distinctive consequences that both the pursuer motion primitives and the motion strategies of both players also change w.r.t. the Homicidal Chauffeur solution.

\section{PROBLEM FORMULATION}

A Differential Drive Robot (DDR), the pursuer, and a omnidirectional evader move on a plane without obstacles. The DDR tries to capture the evader. The game is over when the distance between the DDR and the evader is smaller than a critical value $l$. Both players have maximum bounded speeds $V_{p}^{\max }$ and $V_{e}^{\max }$, respectively. The DDR is faster than the evader, $V_{p}^{\max }>V_{e}^{\max }$, but it can only change its motion direction at a rate that is inversely proportional to its translational speed [5]. We consider here a purely kinematic problem, and neglect any effects due to dynamic constraints (e.g., acceleration bounds). The DDR wants to minimize the capture time $t_{f}$ while the evader wants to maximize it. We want to know the optimal strategies that are in Nash Equilibrium and are used by both players to achieve their goals.

\section{MODEL}

\section{A. Realistic space}

The kinematics of the game can be described in an global coordinate system, $\left(x_{p}, y_{p}, \theta_{p}\right)$ represents the pose of the DDR and $\left(x_{e}, y_{e}\right)$ is the position of the omnidirectional evader. The Euclidean plane is called by Isaacs the realistic space [3]. The state of the system can be expressed as $\left(x_{p}, y_{p}, \theta_{p}, x_{e}, y_{e}\right) \in \mathbb{R}^{2} \times S^{1} \times \mathbb{R}^{2}$. The evolution of the system is described by the following motion equations

$$
\begin{aligned}
& \dot{x}_{p}=\left(\frac{\omega_{1}+\omega_{2}}{2}\right) \cos \theta_{p}, \dot{y}_{p}=\left(\frac{\omega_{1}+\omega_{2}}{2}\right) \sin \theta_{p} \\
& \dot{\theta}_{p}=\left(\frac{\omega_{2}-\omega_{1}}{2 b}\right), \dot{x}_{e}=v_{e} \cos \psi_{e}, \dot{y}_{e}=v_{e} \sin \psi_{e}
\end{aligned}
$$

where $\omega_{1}, \omega_{2} \in\left[-V_{p}^{\max } / r, V_{p}^{\max } / r\right]$ are the controls of the DDR, and they correspond to the angular velocities of its wheels. Let $\omega_{1}$ be the angular velocity of the left wheel and $\omega_{2}$ of the rigth wheel. With a suitable choice of units and assuming an unit radius $r$ of the pursuer's wheels [5], the rotational speeds are equivalent to the translational speed meaning that $V_{p}=\frac{1}{2}\left(\omega_{1}+\omega_{2}\right)$. We denote as $b$ the distance between the center of the robot and the wheel location. The evader controls its speed $v_{e} \in\left[0, V_{e}^{\max }\right]$ and its motion direction using $\psi_{e} \in[0,2 \pi)$. We present two useful definitions for the rest of the paper, $\rho_{v}=V_{e}^{\max } / V_{p}^{\max }$ is the ratio between the maximal translational speed of both players, and $\rho_{d}=b / l$ is the ratio of capture distance $l$ to the distance between the center of the robot and the wheel location $b$. We must have that $l \geq b$, otherwise the capture distance would be located inside the robot.

\section{B. Reduced space}

Usually it is more convenient to analyse the problem and perform all the computations in a space of reduced 
dimension. In our case, the problem can be stated in a coordinate system that is fixed to the body of the DDR (see Fig. 1). The state of the system now can be expressed as $\mathbf{x}=(x, y) \in \mathbb{R}^{2}$. All the orientations in this system are measured with respect to the $y$-axis, in particular, the direction of motion of the evader $\nu$.

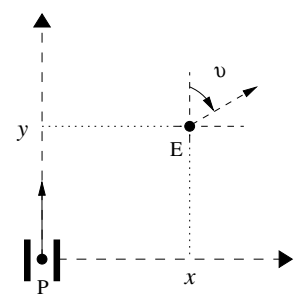

Fig. 1. Reduced space

Using the coordinate transformation given by

$$
\begin{aligned}
& x=\left(x_{e}-x_{p}\right) \sin \theta_{p}-\left(y_{e}-y_{p}\right) \cos \theta_{p} \\
& y=\left(x_{e}-x_{p}\right) \cos \theta_{p}+\left(y_{e}-y_{p}\right) \sin \theta_{p} \\
& \nu=\theta_{p}-\psi_{e}
\end{aligned}
$$

and computing its time derivative, the following model of the kinematics in the DDR-fixed coordinate system is obtained

$$
\begin{aligned}
& \dot{x}=\left(\frac{\omega_{2}-\omega_{1}}{2 b}\right) y+v_{e} \sin \nu \\
& \dot{y}=-\left(\frac{\omega_{2}-\omega_{1}}{2 b}\right) x-\left(\frac{\omega_{1}+\omega_{2}}{2}\right)+v_{e} \cos \nu
\end{aligned}
$$

where $\omega_{1}, \omega_{2} \in\left[-V_{p}^{\max }, V_{p}^{\max }\right]$ are again the controls of the DDR, $v_{e} \in\left[0, V_{e}^{\max }\right]$ is the control associated to the speed of the evader and $\nu \in[0,2 \pi)$ is the control associated to its motion direction in the new coordinate system. Doing $u_{1}=\omega_{1}, u_{2}=\omega_{2}, v_{1}=v_{e}$, and $v_{2}=\nu$, this set of equations can be expressed in the form $\dot{\mathbf{x}}=f(\mathbf{x}, u, v)$, where $u=$ $\left(u_{1}, u_{2}\right) \in \widehat{U}=\left[-V_{p}^{\max }, V_{p}^{\max }\right] \times\left[-V_{p}^{\max }, V_{p}^{\max }\right]$ and $v=$ $\left(v_{1}, v_{2}\right) \in \widehat{V}=\left[0, V_{e}^{\max }\right] \times[0,2 \pi)$.

\section{PRELIMINARIES}

In this section, we describe some concepts from differential games that will be used in the solution of our problem.

\section{A. Value of the game}

The numerical quantity which the players strive to maximize and minimize can assume a variety of forms. However, there is a standard representation of the payoff

$$
J\left(\mathbf{x}_{\mathbf{s}}, u, v\right)=\int_{t_{0}}^{t_{f}} L(\mathbf{x}, u, v) d t+G\left(\mathbf{x}_{\mathbf{f}}\right)
$$

The time integral extends over the path traversed by $\mathbf{x}$ during the game; its lower limit (we could call it $t_{0}$ ) refers to the starting point $\mathbf{x}_{s}$; its upper limit is the time $t_{f}$ to reach the final point $\mathbf{x}_{f} . L(\mathbf{x}, u, v)$ is called the running cost function and it is the cost incurred while the game is being played. The term $G\left(\mathbf{x}_{f}\right)$ is called the terminal cost function and it is the cost incurred for reaching a particular terminal state. For problems of minimum time [1], as in our game, $L(\mathbf{x}, u, v)=$ 1 and $G\left(\mathbf{x}_{f}\right)=0$. Notice that in this case, Eq. (4) does not explicitly depend on time. For a given state of the system $\mathbf{x}_{s}$ at time $t_{0}, V\left(\mathbf{x}_{s}\right)$ represents the outcome if the players implement their optimal strategies starting at the point $\mathbf{x}_{s}$, and it is called the value of the game at $\mathbf{x}_{s}$

$$
V\left(\mathbf{x}_{s}\right)=\min _{u} \max _{v} \int_{t_{0}}^{t_{f}} L(\mathbf{x}, u, v) d t+G\left(\mathbf{x}_{\mathbf{f}}\right)
$$

In our game, Eq. (5) does not explicitly depend on time, and it actually corresponds to the time it takes for the DDR to capture the evader, when the players implement their optimal strategies. No matter what the evader does, it cannot avoid the capture for longer than $V(\mathbf{x})$. If the evader does anything different than its optimal strategy, the DDR can capture it in less than $V(\mathbf{x})$. Reciprocally, if the DDR does anything different than its optimal strategy, the evader can remain longer avoiding it.

\section{B. Equilibrium strategies}

Let $\gamma_{p}(t, \mathbf{x}(t))=u(t)$ and $\gamma_{e}(t, \mathbf{x}(t))=v(t)$ denote the strategies of the DDR and the evader, respectively. A strategy $\operatorname{par}\left(\gamma_{p}^{*}, \gamma_{e}^{*}\right)$ is in (saddle-point) equilibrium if

$$
J\left(\gamma_{p}^{*}, \gamma_{e}\right) \leq J\left(\gamma_{p}^{*}, \gamma_{e}^{*}\right) \leq J\left(\gamma_{p}, \gamma_{e}^{*}\right)
$$

where $J$ is the payoff of the game in terms of the strategies.

\section{Termination situations}

Every state of the system in which the distance between both players equals $l$ represents an opportunity for the DDR to capture the evader. This set of points is called terminal surface [3] or target set [1], and it will be denoted as $\zeta$.

In our game, termination occurs only when the distance between the DDR and the evader is smaller than a critical value $l$ despite any opposition of the evader. The portion of the terminal surface where the DDR can guarantee termination regardless of the choice of controls of the evader is called the usable part (UP) [3]. From [3], we have that the UP of our problem is given by

$$
\mathrm{UP}=\left\{\mathbf{x} \in \zeta: \min _{u \in \widehat{U}} \max _{v \in \widehat{V}} \mathbf{n} \cdot f(\mathbf{x}, u, v)<0\right\}
$$

where $\mathbf{n}$ is the normal vector to $\zeta$ from point $\mathbf{x}$ on $\zeta$ and extending into the playing space. $\mathbf{n} \cdot f(\mathbf{x}, u, v)$ is a projection of the motion directions of both players along the best direction for penetrating $\zeta$ and tell us if the strategies of both players will be able to cross the terminal surface or not. Those points of $\zeta$ where the expression in (7) holds with the inequality reversed are called the non-usable part (NUP) and the game will never terminate on the NUP. The set of points that separates these parts is called the boundary of the usable part (BUP). The BUP can be computed replacing the inequality in (7) by an equality.

\section{NeCESSARY AND SUfFICIENT CONDITIONS FOR SADDle-Point Equilibrium STRATEGIES}

This section describes the necessary and sufficient conditions for existence of saddle-point equilibrium strategies in pursuit-evasion games [1]. 


\section{A. Isaacs equation}

The sufficient condition is provided by an extension of the Hamilton-Jacobi-Bellman (HJB) equation [1] to a noncooperative game with two players. This extension is called the Isaacs equation [1]

$$
-\frac{\partial V(\mathbf{x})}{\partial t}=\min _{u \in \widehat{U}} \max _{v \in \hat{V}}\left[\frac{\partial V(\mathbf{x})}{\partial x} \cdot f(\mathbf{x}, u, v)+L(\mathbf{x}, u, v)\right]
$$

This equation provides a sufficient condition for saddle-point equilibrium strategies.

\section{B. Pontryagin's principle}

$V(\mathbf{x})$ is not known at the beginning of the game thus Eq. (8) cannot directly be used in the derivation of saddle-point equilibrium strategies. An alternative is to use Theorem 1, given below, which is an extension of the Pontryagin's Maximum Principle (PMP) [2] to a two-players non-cooperative game. This extension provides a necessary condition for the existence of saddle-point equilibrium strategies and a constructive manner to compute them. The synthesis of the motion trajectories obtained with Theorem 1 will allow to recover the necessary and sufficient conditions for saddlepoint equilibrium strategies and guarantee global optimality.

Theorem 1 (Pontryagin's Maximum Principle):

Suppose that the pair $\left\{\gamma_{p}^{*}, \gamma_{e}^{*}\right\}$ provides a saddle-point solution in feedback strategies, with $\mathrm{x}^{*}$ denoting the corresponding state trajectory. Furthermore, let its open-loop representation $\left\{u(t)=\gamma_{p}\left(t, \mathbf{x}^{*}(t)\right), v(t)=\gamma_{e}\left(t, \mathbf{x}^{*}(t)\right)\right\}$ also provide a saddle-point solution (in open-loop polices). Then there exists a costate function $p(\cdot):\left[0, t_{f}\right] \rightarrow R^{n}$ such that the following relations are satisfied:

$$
\begin{gathered}
\dot{\mathbf{x}}^{*}(t)=f\left(\mathbf{x}(t), u^{*}(t), v^{*}(t)\right), \dot{\mathbf{x}}^{*}(0)=\mathbf{x}_{s} \\
H(p, \mathbf{x}, u, v)=p^{T} \cdot f(\mathbf{x}, u, v)+L(\mathbf{x}, u, v) \\
H\left(p, \mathbf{x}^{*}, u^{*}, v\right) \leq H\left(p, \mathbf{x}^{*}, u^{*}, v^{*}\right) \leq H\left(p, \mathbf{x}^{*}, u, v^{*}\right) \\
\dot{p}^{T}(t)=-\frac{\partial}{\partial x} H\left(p(t), \mathbf{x}^{*}(t), u^{*}, v^{*}\right) \\
p^{T}(t)=\frac{\partial}{\partial x} G\left(\mathbf{x}_{\mathbf{f}}\right) \text { along } \zeta=0
\end{gathered}
$$

where $T$ denotes the transpose operator.

Equation (12) is known as the adjoint equation, and Eq. (10) as the Hamiltonian function. Using Eq. (10) with $p(t)=$ $\nabla V(\mathbf{x}(t))$ for the case of vector-valued functions, assuming that the Hamiltonian is separable in $u$ and $v$, and that $V(\mathbf{x})$, $f(\mathbf{x}, u, v)$ and $L(\mathbf{x}, u, v)$ do not explicitly depend on time, we can rewrite the Isaacs equation as

$$
\min _{u \in \widehat{U}} \max _{v \in \bar{V}} H(\nabla V(\mathbf{x}), \mathbf{x}, u, v)=0
$$

The vector $\nabla V(\mathbf{x})$ can be interpreted as the Lagrange multipliers used in constrained optimization or optimal control theory.

\section{Vi. Optimal Trajectories}

In this section, we will derive the saddle-point equilibrium strategies for both players.

\section{A. Computing the UP and its boundary}

We will compute the portion of the space where the pursuer guarantees termination regardless of the choice of controls of the evader.

Lemma 1: In this game, the UP has two regions:

1) The first region corresponds to capture the evader when the DDR is moving forward following a straight line in the realistic space. This region contains all the points on $\zeta$ such that $\cos s>\rho_{v}$ and its boundary is given by those points where $\cos s=\rho_{v}$.

2) The second region corresponds to capture the evader when the DDR is moving backward following a straight line in the realistic space. This region contains all the points on $\zeta$ such that $\cos s<-\rho_{v}$ and its boundary is given by those points where $\cos s=-\rho_{v}$.

Proof: For this problem, the terminal surface $\zeta$ is characterized by the distance $l$ between both players. In the reduced space, $\zeta$ is a circle of radius $l$ centred at the origin, hence we can parametrise it by the angle $s$, which is the angle between the evader's position and the pursuer's heading at the end of the game (recall that all the orientations in the reduced space are measured with respect to the $y$-axis). The game terminates with

$$
x=l \sin s, y=l \cos s
$$

The outward normal $\mathbf{n}$ to $\zeta$ is defined by

$$
\mathbf{n}=\left[\begin{array}{ll}
\sin s & \cos s
\end{array}\right]
$$

The UP after substituting Eq. (3) and Eq. (16) into inequality (7) is given by

$$
\begin{aligned}
& \min _{u_{1}, u_{2}} \max _{v_{1}, v_{2}}\left\{\sin s\left[\left(\frac{u_{2}-u_{1}}{2 b}\right) y+v_{1} \sin v_{2}\right]\right. \\
& \left.+\cos s\left[-\left(\frac{u_{2}-u_{1}}{2 b}\right) x-\left(\frac{u_{1}+u_{2}}{2}\right)+v_{1} \cos v_{2}\right]\right\}<0
\end{aligned}
$$

Substituting Eq. (15) into inequality (17) and after doing some algebra, we found that

$$
\min _{u_{1}, u_{2}}\left[V_{e}^{\max }-\left(\frac{u_{1}+u_{2}}{2}\right) \cos s\right]<0
$$

In inequality (18) we have two cases, (1) $\cos s>0$ or (2) $\cos s<0$. In order to make inequality (18) minimal, $u_{1}$ and $u_{2}$ must be equal and saturated (that is equal to $\left|V_{p}^{\max }\right|$ ). Hence the pursuer moves in straight line. If $\cos s>0$ then $\left(\frac{u_{1}+u_{2}}{2}\right)>0$, the DDR is moving forward and if $\cos s<0$ then $\left(\frac{u_{1}+u_{2}}{2}\right)<0$, the DDR is moving a backward. Note that the same controls $u_{1}$ and $u_{2}$ are used in both the reduced and realistic spaces. From inequality 18 and considering the two cases described above, it is straightforward to compute that the region where the DDR is moving forward contains all points such that $\cos s>\rho_{v}$ and the region where the DDR is moving backwards contains all the points such that $\cos s<-\rho_{v}$.

\section{B. Hamiltonian}

In our problem, we have that $\nabla V=\left[\begin{array}{ll}V_{x} & V_{y}\end{array}\right]^{T}$ and $L\left(\mathbf{x}, u_{1}, u_{2}, v_{1}, v_{2}\right)=1 . V_{x}$ and $V_{y}$ represent the partial derivatives $\frac{\partial V}{\partial x}$ and $\frac{\partial V}{\partial y}$. Substituting the last expressions and the motion equations in (3) into Eq. (10), we obtain 


$$
\begin{aligned}
& H\left(\mathbf{x}, \nabla V, u_{1}, u_{2}, v_{1}, v_{2}\right)=V_{x}\left(\frac{u_{2}-u_{1}}{2 b}\right) y+V_{x} v_{1} \sin v_{2} \\
& -V_{y}\left(\frac{u_{2}-u_{1}}{2 b}\right) x-V_{y}\left(\frac{u_{1}+u_{2}}{2}\right)+V_{y} v_{1} \cos v_{2}+1
\end{aligned}
$$

Lemma 2: The Hamiltonian of our system is separable in the controls of the pursuer and the evader, i.e., we can write it in the form $f_{1}(\mathbf{x}, \nabla V, u)+f_{2}(\mathbf{x}, \nabla V, v)$.

Proof: In our case, Eq. (19) can be rewritten in the form $H\left(\mathbf{x}, \nabla V, u_{1}, u_{2}, v_{1}, v_{2}\right)=\frac{u_{1}}{2}\left(\frac{-y V_{x}}{b}+\frac{x V_{y}}{b}-V_{y}\right)+$ $\frac{u_{2}}{2}\left(\frac{y V_{x}}{b}-\frac{x V_{y}}{b}-V_{y}\right)+v_{1}\left(V_{x} \sin v_{2}+V_{y} \cos v_{2}\right)+1$.

\section{Optimal controls}

Lemma 3: The time-optimal controls for the DDR that satisfy the Isaacs' equation (8) are given by

$$
\begin{aligned}
& u_{1}^{*}=-\operatorname{sgn}\left(\frac{-y V_{x}}{b}+\frac{x V_{y}}{b}-V_{y}\right) V_{p}^{\max } \\
& u_{2}^{*}=-\operatorname{sgn}\left(\frac{y V_{x}}{b}-\frac{x V_{y}}{b}-V_{y}\right) V_{p}^{\max }
\end{aligned}
$$

We have that both controls are always saturated. The controls of the evader are given by

$$
v_{1}^{*}=V_{e}^{\max }, \sin v_{2}^{*}=\frac{V_{x}}{\rho}, \cos v_{2}^{*}=\frac{V_{y}}{\rho}
$$

where $\rho=\sqrt{V_{x}^{2}+V_{y}^{2}}$. The evader moves at maximal speed.

Proof: By Lemma 2 we know that the Hamiltonian of our game is separable in two parts, one in terms of the pursuer's controls and other in terms of the evader's controls. Let consider first the pursuer and later the evader. As the DDR is the minimizer player it wants the Hamiltonian term $\frac{u_{1}}{2}\left(\frac{-y V_{x}}{b}+\frac{x V_{y}}{b}-V_{y}\right)+\frac{u_{2}}{2}\left(\frac{y V_{x}}{b}-\frac{x V_{y}}{b}-V_{y}\right)$ to be minimal. Let $A=\frac{-y V_{x}}{b}+\frac{x V_{y}}{b}-V_{y}$ and $B=\frac{y V_{x}}{b}-\frac{x V_{y}}{b}-V_{y}$. There are four cases, in which $u_{1}$ and $u_{2}$ must be saturated and they correspond to the maximal translational speed $V_{p}^{\max }$.

1) If $A<0$ and $B<0$ then $u_{1}=V_{p}^{\max }$ and $u_{2}=V_{p}^{\max }$, the pursuer moves forward in straight line.

2) If $A>0$ and $B>0$ then $u_{1}=-V_{p}^{\max }$ and $u_{2}=$ $-V_{p}^{\max }$, the pursuer moves backward in straight line.

3) If $A>0$ and $B<0$ then $u_{1}=-V_{p}^{\max }$ and $u_{2}=$ $V_{p}^{\max }$, the pursuer rotates in place counterclockwise.

4) If $A<0$ and $B>0$ then $u_{1}=V_{p}^{\max }$ and $u_{2}=$ $-V_{p}^{\max }$, the pursuer rotates in place clockwise.

Analogously, as the evader is the maximizer player it wants the term $v_{1}\left(V_{x} \sin v_{2}+V_{y} \cos v_{2}\right)$ to be maximal. The quantity in round parenthesis is the dot product of the vectors $\left[\begin{array}{ll}V_{x} & V_{y}\end{array}\right]$ and $\left[\sin v_{2} \cos v_{2}\right]$, and it is maximal when $\left[\sin v_{2} \quad \cos v_{2}\right]$ lies along $\left[\begin{array}{ll}V_{x} & V_{y}\end{array}\right]$ (both vectors are parallel and have the same direction). Hence $v_{1}=V_{e}^{\max }$ and $\left[V_{x} V_{y}\right] \|\left[\sin v_{2} \cos v_{2}\right]$.

In Lemma 9, we will present the actual evader trajectories.

\section{Adjoint equation}

If $t_{f}$ is the time of termination of the game, we define the retro-time as $\tau=t_{f}-t$. The adjoint equation in its retro-time form is

$$
\frac{d}{d \tau} \nabla V[\mathbf{x}(\tau)]=\frac{\partial}{\partial x} H\left(\mathbf{x}, \nabla V, u_{1}^{*}, u_{2}^{*}, v_{1}^{*}, v_{2}^{*}\right)
$$

We integrate the adjoint equation backward in time from the UP to obtain $\nabla V(\mathbf{x})$. Substituting $\nabla V(\mathbf{x})$ into the optimal controls expressions and the resulting controls into the motion equations leads to the optimal trajectories starting at UP.

Lemma 4: The expressions in retro-time of the adjoint equation of our system are

$$
\frac{d}{d \tau} V_{x}=-\left(\frac{u_{2}^{*}-u_{1}^{*}}{2 b}\right) V_{y}, \frac{d}{d \tau} V_{y}=\left(\frac{u_{2}^{*}-u_{1}^{*}}{2 b}\right) V_{x}
$$

Proof: Substituting Eq. (19) into Eq. (22) ( $u_{1}^{*}, u_{2}^{*}, v_{1}^{*}$ and $v_{2}^{*}$ denote the optimal controls of both players) it is straightforward to obtain the expressions above.

In what follows, we will show that the players' optimal motion primitives in the realistic space correspond for the evader to straight lines (see Lemmas 6 and 9), and for the pursuer to rotations in place and straight lines, Lemma 7.

\section{E. Integrating the adjoint equation starting at the UP}

We need to establish the initial conditions of the system, in this case, the values of $V_{x}$ and $V_{y}$ on the UP of $\zeta$. From Eq. (15), we have that

$$
\frac{d x}{d s}=l \cos s, \frac{d y}{d s}=-l \sin s
$$

Since $V(\mathbf{x})=0$ on the UP of $\zeta$ it follows that

$$
V_{s}=\frac{d V}{d s}=\frac{\partial V}{\partial x} \frac{d x}{d s}+\frac{\partial V}{\partial y} \frac{d y}{d s}=0
$$

Substituting Eq. (24) into Eq. (25), we obtain $V_{x} \cos s=$ $V_{y} \sin s$. From this expression, we have that on the UP

$$
V_{x}=\lambda \sin s, V_{y}=\lambda \cos s
$$

where $\lambda$ is a constant value.

Lemma 5: The solution of the adjoint equation (23) starting at the UP is

$$
V_{x}=\lambda \sin s, V_{y}=\lambda \cos s
$$

Proof: From Lemma 1, we know that near the end of game the DDR is following a translation. Therefore Eq. (23) takes the form $\frac{d}{d \tau} V_{x}=0, \frac{d}{d \tau} V_{y}=0$. We can directly verify that Eq. (27) satisfies the last expressions.

Lemma 6: The evader is following a straight line in the reduced and realistic spaces near the end of the game.

Proof: From Eq. (27), we know that $V_{x}$ and $V_{y}$ have constant values. Substituting those values into the evader's controls in Eq. (21), we found that $\nu=v_{2}^{*}=s$, the evader's motion direction in the reduced space, is also constant, thus the evader is following a straight line in the reduced space near the end of the game. From Lemma 1, we know that the DDR is moving in straight line near the end of the game. Therefore its motion direction $\theta_{p}$ is constant. From the third expression in Eq. (2), and as $\nu$ and $\theta_{p}$ are constant, it is straightforward to see that $\psi_{e}$, the evader's motion direction in the realistic space, will be constant.

Remark 1: From Lemma 3, the controls of the players are independent, it would be misleading to conclude that Lemma 6 implies that the evader's controls depend on the pursuer's controls. But in order to show a graphical representation of the trajectories in the realistic space it is 
necessary to know the controls of the DDR to compute the transformation between the reduced and realistic spaces.

\section{$F$. Integrating the motion equations starting at the UP}

Theorem 2: The trajectories of the system in the reduced space leading directly to the end of the game are

$$
\begin{aligned}
& x=-\tau V_{e}^{\max } \sin s+l \sin s \\
& y=\tau\left(-V_{e}^{\max } \cos s \pm V_{p}^{\max }\right)+l \cos s
\end{aligned}
$$

the sign + is taken if the DDR is translating forward when it captures the evader and the sign - if it is translating backward.

Proof: The retro-time version of the motion equations in the reduced space is

$$
\begin{aligned}
& \frac{d}{d \tau} x=-\left(\frac{u_{2}-u_{1}}{2 b}\right) y-v_{1} \sin v_{2} \\
& \frac{d}{d \tau} y=+\left(\frac{u_{2}-u_{1}}{2 b}\right) x+\left(\frac{u_{1}+u_{2}}{2}\right)-v_{1} \cos v_{2}
\end{aligned}
$$

Substituting Eq. (27) into the controls expressions in Eq. (20) and Eq. (21), and the resulting expressions into Eq. (29) we obtain $\frac{d}{d \tau} x=-V_{e}^{\max } \sin s, \frac{d}{d \tau} y=-V_{e}^{\max } \cos s \pm V_{p}^{\max }$ the sign + is taken if the DDR is translating forward, and the sign - if it is translating backward. Integrating the last equations with the initial conditions $x=l \sin s$ and $y=$ $l \cos s$ leads to the expressions in Eq. (28) for the trajectories.

\section{G. Transition surface}

The solutions in Eq. (27), and Eq. (28) are valid as long as the DDR does not switch controls. The place where a control variable abruptly changes in value, is known as a transition surface. In our problem, after a time interval the DDR switches controls and it starts rotating in place in the realistic space.

Lemma 7: The DDR switches controls and it starts a rotation in place in the realistic space, at $\tau_{s}=\left|\frac{b \cos s}{V_{p}^{\max } \sin s}\right|$. If $s \in[0, \pi], u_{2}^{*}$ switches first, otherwise, $u_{1}^{*}$.

Proof: We can compute the time $\tau_{s}$ when the DDR switches controls, substituting Eq. (27) and Eq. (28) into Eq. (20), and verifying which one of the resulting expressions is the first in changing signs. Doing that we find that for $s \in\left[0, \frac{\pi}{2}\right], u_{2}^{*}$ switches first and it do it at

$$
\tau_{s}=\frac{b \cos s}{V_{p}^{\max } \sin s}
$$

The other cases can be proved using an analogous reasoning.

When the game reaches $\tau_{s}$, we need to start a new integration of the retro-time version of the adjoint (23) and motion (29) equations. This integration takes as initial conditions the values of $V_{x}, V_{y}, x$, and $y$ at $\tau_{s}$. We will denote those values as $V_{x_{\tau_{s}}}, V_{y_{\tau_{s}}}, x_{\tau_{s}}$ and $y_{\tau_{s}}$. The equations in Lemmas 8 and 9, and Theorem 3 were constructed after the DDR switches controls and it starts rotating in place in the realistic space.
Lemma 8: The solution of the adjoint equation (23) start-

$$
\begin{array}{r}
\text { ing at } \tau_{s} \text { is } V_{x}=\lambda \sin \left[s-\left(\frac{u_{2}^{*}-u_{1}^{*}}{2 b}\right)\left(\tau-\tau_{s}\right)\right] \\
V_{y}=\lambda \cos \left[s-\left(\frac{u_{2}^{*}-u_{1}^{*}}{2 b}\right)\left(\tau-\tau_{s}\right)\right] \\
\text { for } \tau \geq \tau_{s} \text {. }
\end{array}
$$

Proof: Computing the retro-time derivative of Eq. (23), we obtain two ordinary linear differential equations of second order with constant coefficients $\frac{d^{2}}{d \tau^{2}} V_{x}=$ $-\left(\frac{u_{2}^{*}-u_{1}^{*}}{2 b}\right)^{2} V_{x}, \frac{d^{2}}{d \tau^{2}} V_{y}=-\left(\frac{u_{2}^{*}-u_{1}^{*}}{2 b}\right)^{2} V_{y}$. Solving these equations with the values of $V_{x}$ and $V_{y}$ at $\tau_{s}$ as initial conditions we obtain the expressions in Eq. (31).

Lemma 9: The evader is following a straight line in the realistic space while the $D D R$ is rotating in place.

Proof: Substituting Eq. (31) into Eq. (21) we have that, the evader's motion direction $\nu^{\prime}=v_{2}^{*}=s-\left(\frac{u_{2}^{*}-u_{1}^{*}}{2 b}\right)(\tau-$ $\left.\tau_{s}\right)$. As the DDR is rotating in place, its motion direction is given by $\theta_{p}^{\prime}=\theta_{p}^{s}-\left(\frac{u_{2}^{*}-u_{1}^{*}}{2 b}\right)\left(\tau-\tau_{s}\right)$ where $\theta_{p}^{s}$ is the initial motion direction of the DDR in the realistic space. Substituting $\nu^{\prime}$ and $\theta_{p}^{\prime}$ into the third expression in Eq. (2) we obtain that $\psi_{e}=\theta_{p}^{s}-s$, the evader's motion direction in the realistic space. Note that it is a constant value, thus the evader is following a straight line in the realistic space.

Theorem 3: The trajectories of the system in the reduced space starting at $\tau_{s}$ are

$$
\begin{aligned}
& x=-y_{\tau_{s}} \sin \left[\left(\frac{u_{2}^{*}-u_{1}^{*}}{2 b}\right)\left(\tau-\tau_{s}\right)\right]+x_{\tau_{s}} \cos \left[\left(\frac{u_{2}^{*}-u_{1}^{*}}{2 b}\right)\left(\tau-\tau_{s}\right)\right] \\
& -\left(\tau-\tau_{s}\right) V_{e}^{\max } \sin \left[s-\left(\frac{u_{2}^{*}-u_{1}^{*}}{2 b}\right)\left(\tau-\tau_{s}\right)\right] \\
& y=x_{\tau_{s}} \sin \left[\left(\frac{u_{2}^{*}-u_{1}^{*}}{2 b}\right)\left(\tau-\tau_{s}\right)\right]+y_{\tau_{s}} \cos \left[\left(\frac{u_{2}^{*}-u_{1}^{*}}{2 b}\right)\left(\tau-\tau_{s}\right)\right] \\
& -\left(\tau-\tau_{s}\right) V_{e}^{\max } \cos \left[s-\left(\frac{u_{2}^{*}-u_{1}^{*}}{2 b}\right)\left(\tau-\tau_{s}\right)\right]
\end{aligned}
$$

Proof: Substituting Eq. (31) into Eq. (21), and the resulting expressions into Eq. (29) we obtain $\frac{d}{d \tau} x=$ $-\left(\frac{u_{2}^{*}-u_{1}^{*}}{2 b}\right) y-V_{e}^{\max } \sin \left[s-\left(\frac{u_{2}^{*}-u_{1}^{*}}{2 b}\right)\left(\tau-\tau_{s}\right)\right], \frac{d}{d \tau} y=$ $\left(\frac{u_{2}^{*}-u_{1}^{*}}{2 b}\right) x-V_{e}^{\max } \cos \left[s-\left(\frac{u_{2}^{*}-u_{1}^{*}}{2 b}\right)\left(\tau-\tau_{s}\right)\right]$. Computing the retro-time derivative of these expressions and solving the resulting expressions with the initial conditions $x_{\tau_{s}}$ and $y_{\tau_{s}}$ we obtain the solution in Eq. (32).

Note that in Eqs. (31) and (32), $\left(u_{1}^{*}, u_{2}^{*}\right)$ only takes the values $\left(-V_{p}^{\max }, V_{p}^{\max }\right)$ and $\left(V_{p}^{\max },-V_{p}^{\max }\right)$ corresponding to a rotation in place of the DDR in the realistic space.

\section{DECISION PROBLEM}

A game of kind is a game in which we are interested in what conditions lead to a winning for each one of the players. In our case, this corresponds to find the conditions that make capture possible for the DDR or escape for the evader.

\section{A. The barrier}

There is a surface called the barrier [3], which separates the set of starting positions in those that result in capture and those that result in escape for the evader. The answer to the capture-escape question relies on whether or not the barrier divides the playing space into two parts. 


\section{B. Construction of the barrier}

As the BUP separates the points on $\zeta$ where immediate capture occurs from those where does not, it is used as initial condition for the barrier. The barrier is constructed integrating the adjoint and motion equations starting at the BUP. Suppose the barrier separates the playing space into two parts. If $\mathbf{x}$ is in the outer side, the one that is not contiguous to the UP, then the DDR cannot force the capture because the UP is not accessible. If the barrier fails to separate the playing space, then capture can always be attained by the DDR.

\section{Symmetry of the problem}

Figure 2 shows a representation of the terminal surface $\zeta$, the UP and its boundary in the reduced space. The system exhibits some symmetries with respect to the $x$ and $y$-axis in this representation. An analysis for the trajectories in the first quadrant will be provided. This analysis can be extended to the remaining quadrants using an analogous reasoning.

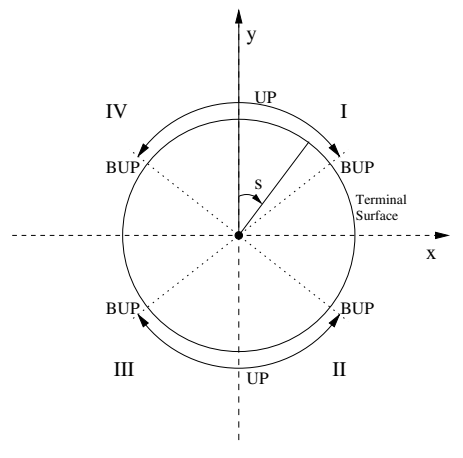

Fig. 2. Representation of $\zeta$, UP and its boundary in the reduced space.

\section{Solving the decision problem}

We present two useful properties appearing in some trajectories reaching the UP.

Lemma 10: The retro-time trajectories starting at the UP in the first quadrant (see Fig. 2) reach the $y$-axis before the system switches controls if $l / V_{e}^{\max } \leq \tau_{s}$.

Proof: When the retro-time trajectories reach the $y$-axis we have that $x=0$. From Eq. (28), $-\tau V_{e}^{\max } \sin s+l \sin s=$ 0 . By straightforward algebraic manipulation, we find that $\tau=\tau_{c}=l / V_{e}^{\max }$. This is the retro-time it takes to reach the $y$-axis if the system is following Eq. (28). We know that the DDR switches controls at $\tau=\tau_{s}$, if $\tau_{c} \leq \tau_{s}$ the system will reach the $y$-axis before switching controls.

Lemma 11: The trajectories in Eq. (28) that reach the $y$-axis in the first quadrant, reach it at $y=l / \rho_{v}$.

Proof: From Lemma 10, we have that $\tau_{c}=l / V_{e}^{\max }$ is the retro-time it takes to reach the $y$-axis when the system is following Eq. (28). Substituting $\tau_{c}$ into Eq. (28) we have that $y=\left(l / V_{e}^{\max }\right)\left(-V_{e}^{\max } \cos s+V_{p}^{\max }\right)+l \cos s$. After straightforward algebraic manipulation, we find that $y=y_{c}=l / \rho_{v}$.
Lemma 12: The barrier consists of a straight line segment, and it reaches the $y$-axis in the first quadrant if $\rho_{v} \geq|\tan S| / \rho_{d}$ where $S=\cos ^{-1}\left(\rho_{v}\right)$ is the angle at the BUP (see Fig. 2).

Proof: In our game, the barrier is constructed by substituting the value $S=\cos ^{-1}\left(\rho_{v}\right)$ into Eq. (28). After a retro-time interval $\tau_{s}$ the DDR should switch controls and start rotating in place in the realistic space. Then the system should follow the trajectory described by Eq. (32) in the reduced space. Figure 3 shows both trajectories. The trajectory given by Eq. (32) intersects the initial segment of the barrier and it comes back to the UP in the reduced space. According to [3], the barrier is not crossed by any trajectory followed by the system during optimal play, in particular, it cannot cross itself. Also, it is possible to show that the points over the portion of the trajectory given by Eq. (32) correspond to states where the DDR can attain capture, which contradicts the neutral nature of the barrier. Therefore the portion of the trajectory given by Eq. (32), (the arc in Fig. 3) must be discarded. The barrier reaches the terminal surface with $S=\cos ^{-1}\left(\rho_{v}\right)$, and it consists only of a straight line in the reduced space given by Eq. (28) that ends when $\tau=\tau_{s}$. From Lemma 10, it is straightforward to verify that the barrier will reach the $y$-axis if $\tau_{c} \leq \tau_{s}$. Substituting the values of $\tau_{c}$ and $\tau_{s}$ in the last inequality, we find that it can be expressed as $V_{e}^{\max } / V_{p}^{\max } \geq(l|\tan S|) / b$, which can be rewritten as $\rho_{v} \geq|\tan S| / \rho_{d}$.

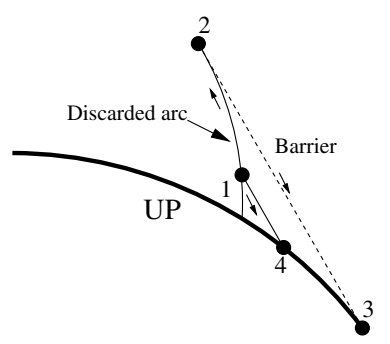

Fig. 3. The system is following the barrier from point 1 to point 3 . The two elements of the barrier are, the dashed line which is given by Eq. (28), and the solid arc given by Eq. (32). The solid straight line represents the optimal trajectory to reach the UP at point 4 from point 1 . From points over the solid arc the DDR can attain capture and the system follows a straight line in the reduced space. Therefore this arc must be discarded.

Theorem 4: If $\rho_{v}<|\tan S| / \rho_{d}$ the DDR can capture the evader from any initial configuration in the playing space. Otherwise the barrier separates the space into two regions:

1) One between the UP and the barrier.

2) Another above the barrier.

The DDR can only force the capture in the configurations between the UP and the barrier, in which case, the DDR follows a straight line in the realistic space when it captures the evader.

Proof: It follows from the definition of the barrier and Lemma 12. All the trajectories between the barrier and the UP are straight lines reaching the $y$-axis (refer to Lemma 11 and see Fig. 4). 


\section{PARTITION OF THE SPACE}

A procedure commonly used to obtain the optimal controls generating the optimal trajectories for the players in the entire state space is to split this space into a set of mutually disjoint regions. In each one of them, $V(\mathbf{x})$ is continuously differentiable. The behaviour and the construction of $V(\mathbf{x})$ is well established in such regions. The boundaries of these regions are called singular surfaces [3], [12], [1], and $V(\mathbf{x})$ is not continuously differentiable across them.

\section{A. Singular surface}

Following the definition given in [1], a singular surface is a manifold on which

1) the equilibrium strategies are not uniquely determined by the necessary conditions of Theorem 2 or

2) the $V(\mathbf{x})$ is not continuously differentiable, or

3) the $V(\mathbf{x})$ is discontinuous.

1) Transition surface (TS): The place where a control variable abruptly changes in value, is known as a transition surface (see Lemma 15 for its construction in our game).

2) Universal surface (US): A surface to which optimal trajectories enter from both sides -called the tributary trajectories- and then stay on, is called a universal surface. (see Lemma 16 for their construction in our game).

3) Dispersal surface $(D S)$ : A dispersal surface is defined in [3], [1] as the locus of initial conditions along which the strategy of the players are not unique. In our game it appears due to symmetries in the reduced space, see subsection VIII$\mathrm{C}$ and Fig. 4.

Note that in our game we have found these three types of singular surfaces. However there are other known types in the literature [1].

\section{B. Regions in the space}

In this section, we describe the regions of the space characterized by a particular combination of the motion primitives of both players.

Lemma 13: The trajectories reaching the $y$-axis in the first quadrant have an orientation $s \in\left[0, \tan ^{-1}\left(\rho_{v} \rho_{d}\right)\right]$ at the UP (refer to Fig. 2).

Proof: From Lemma 10, the trajectories that reach the $y$-axis are those that $\tau_{c} \leq \tau_{s}$. The last one that can reach it will have $\tau_{c}=\tau_{s}$. Substituting the corresponding values $l / V_{e}^{\max }=(b \cos s) /\left(V_{p}^{\max } \sin s\right)$. From the last expression we find that $\tan s=\rho_{v} \rho_{d}$. The trajectory given by $s=0$ coincides with the $y$-axis. Therefore, the trajectories crossing the $y$-axis will have an angle $s \in\left[0, \tan ^{-1}\left(\rho_{v} \rho_{d}\right)\right]$ at the UP.

Lemma 14: The trajectories that have an orientation $s \in$ $\left(\tan ^{-1}\left(\rho_{v} \rho_{d}\right), \cos ^{-1}\left(\rho_{v}\right)\right]$ in the UP of the first quadrant terminate when the DDR switches controls.

Proof: From Lemma 13 we know that the last trajectory reaching the $y$-axis have an orientation $s_{c}=\tan ^{-1}\left(\rho_{v} \rho_{d}\right)$. If $s>s_{c}$ the DDR switches controls before reaching the $y$-axis and the system starts following the trajectories given by Eq. (32). The value $s=\cos ^{-1}\left(\rho_{v}\right)$ corresponds to the barrier and it only consists of a straight line. Thus the straight line trajectories reaching the UP at $s \in\left(\tan ^{-1}\left(\rho_{v} \rho_{d}\right), \cos ^{-1}\left(\rho_{v}\right)\right]$ terminate when a switch of the DDR controls occurs.

Lemma 15: The points $\mathrm{x}$ in the reduced space where $\tau=$ $\tau_{s}$ constitute a TS in the first quadrant. At the TS in the first quadrant, the expression $y V_{x}-x V_{y}-b V_{y}=0$ is satisfied. This surface is bounded by the barrier and the y-axis.

Proof: From the Lemma 14, we know that the trajectories ending at $s \in\left(\tan ^{-1}\left(\rho_{v} \rho_{d}\right), \cos ^{-1}\left(\rho_{v}\right)\right)$ have a switch when $\tau=\tau_{s}$. The points $\mathbf{x}$ where this happen constitute the transition surface (TS). For the first quadrant, in those points $u_{2}$ changes sign. If $s=\cos ^{-1}\left(\rho_{v}\right)$, the trajectory corresponds to the barrier, which is a straight line ending also just before switching controls. Thus the $y$-axis and the barrier bound the TS.

Lemma 16: The positive $y$-axis contains a US where the pursuer follows the evader with its heading directly aligned to it.

Proof: A necessary condition for a US, is that in this surface there are no switches and the controls of the players remain constant. From Lemma 13, one time-optimal trajectory for the system corresponds to a straight line with $s=0$, i.e., the evader's relative position aligned with the pursuer's heading. From Lemma 5, we know that along this trajectory starting at UP and reaching $y_{c}, V_{x}=\lambda \sin s$, $V_{y}=\lambda \cos s$ where $\lambda$ is a constant value. At $y_{c}$ and as $s=0$, $V_{x}=0, V_{y}=\lambda$. Substituting those values into the pursuer's controls Eq. (20), we find that the expressions inside the sign functions are constant. Therefore, the DDR does not need to switch controls. Substituting $V_{x}=0, V_{y}=\lambda$ into the evader's controls Eq. (21), we find that its motion direction is also constant. Hence, the system is moving in straight line over the $y$-axis in the reduced space. In the realistic space, both players are moving following a straight line while the system is moving over the positive $y$-axis in the reduced space.

Lemma 17: The tributary trajectories reaching the US associated to the first quadrant correspond to a rotation in place of the DDR and straight line for the evader in the realistic space.

Proof: In our game, the tributary trajectories entering the US are generated by a different combination of the optimal controls for the DDR (see Lemmas 6 and 9). For the first quadrant, we have that $u_{1}^{*}=V_{p}^{\max }$ and $u_{2}^{*}=-V_{p}^{\max }$ (the DDR rotates clockwise to align its heading with the evader's motion direction in the realistic space). Taking these controls, the trajectories in the reduced space can be computed using an analogous reasoning to the one applied in Theorem 3.

\section{Construction of the partition of the space}

In Fig. 4, we present the partition and the corresponding trajectories in the reduced space.

1) Region I: It is the set of points (shown in Fig. 4) that can reach the UP with a single straight line trajectory in the reduced space, which corresponds to a straight line motion of both, the DDR and the evader, in the realistic space. 
2) Region II: It is the set of points (shown in Fig. 4) that reach the TS by following a trajectory given by Eq. (32) in the reduced space, which corresponds to a rotation in place for DDR and straight line trajectory for the evader, both in the realistic space.

3) Region III: It is the set of points (shown in Fig. 4) that reach the US following one of its tributary trajectories given by Eq. (32) in the reduced space corresponding to a rotation in place for the DDR and a straight line trajectory for the evader, both in the realistic space.

In this figure, the $x$-axis contains a dispersal surface (DS), represented as a bold line, where the rotation in place trajectories coming from the upper and bottom parts of the UP intersect. Over the DS the DDR have the option to rotate either clockwise or counterclowise to catch the evader, both trajectories will lead to the same optimal time-to-go. The difference will be that at the end the capture will be attained moving forward or backward in straight line in the realistic space.

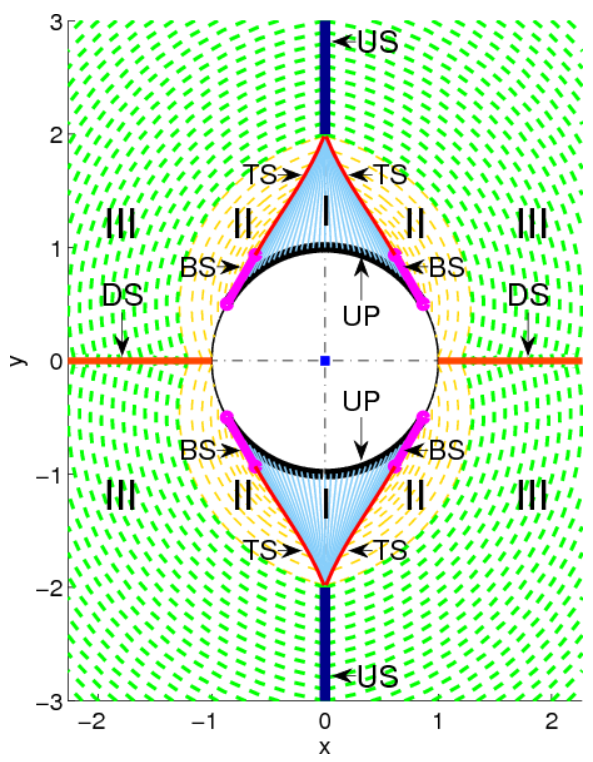

Fig. 4. Partition of the reduced space.

\section{Simulations}

In this section, we present simulation results of the optimal motion primitives of both players and the trajectories of the system. The parameters used were $V_{p}^{\max }=1.0, V_{e}^{\max }=0.5$, $b=1.0$ and $l=1.0$. At the beginning of the game (see. Fig. 5 ), the DDR is located at $P_{I}$ and the evader at $E_{I}$. The capture condition is achieved when the players are at the positions $P_{F}$ and $E_{F}$, respectively. In this figure, we can observe that the DDR first rotates in place at $P_{I}$ aligning its heading with the point $P_{F}$, once it has accomplished that, it moves toward the point $P_{F}$ following a forward motion in straight line. The evader moves from point $E_{I}$ to point $E_{F}$ following a forward motion in straight line. In the reduced space (see Fig. 4), the system follows a trajectory in region
II reaching the TS. At that point, it follows a trajectory in region I reaching the terminal surface.

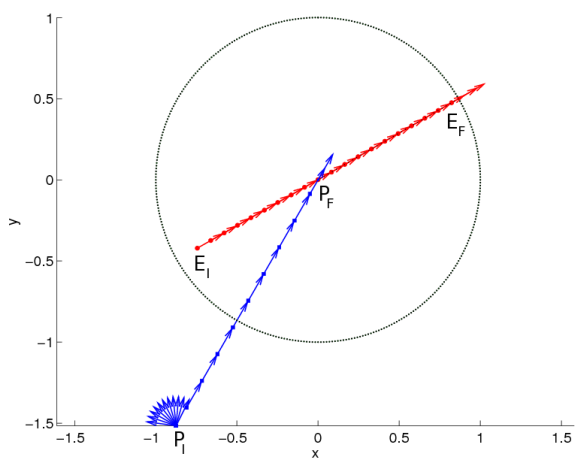

Fig. 5. The DDR captures the evader rotating in place and then it follows a forward motion in straight line.

\section{Conclusions And Future Work}

In this paper, we found closed-form representations of the motion primitives and time-optimal controls for each player. In the realistic space, the motion primitives for the pursuer are straight lines and rotations in place and for the evader are straight lines. The strategies of the players that we have found are in Nash Equilibrium. We also construct a solution for the entire reduced space, and we present the conditions defining the winner of the game. As future work, we want to include acceleration bounds in the solution of the problem.

\section{REFERENCES}

[1] T. Başar and G. Olsder, Dynamic Noncooperative Game Theory, 2nd $E d$. SIAM Series in Classics in Applied Mathematics, Philadelphia, 1999.

[2] L. S. Pontryagin, V. G. Boltyanskii, R. V. Gamkrelidze, and E. F. Mishchenko. The Mathematical Theory of Optimal Processes. JohnWiley, 1962.

[3] R. Isaacs. Differential Games. Wiley, New York, 1965.

[4] S. Bhattacharya and S. Hutchinson, On the existence of nash equilibrium for a two player pursuit-evasion game with visibility constraints. International Journal on Robotics Research, Dec. 2009.

[5] D.J. Balkcom and M.T. Mason, Time Optimal Trajectories for Bounded Velocity Differential Drive Vehicles, International Journal of Robotics Research Vol 21, No 3, pp 219-232, 2002.

[6] L. Guibas, J.-C. Latombe,S.M. LaValle, D. Lin, R. Motwani, Visibility-based pursuit-evasion in a polygonal environment, International Journal of Computational Geometry and Applications, 9(5):471-494, 1999.

[7] T. Bandyopadhyay, Y. Li, M.H. Ang and D. Hsu, A Greedy Strategy for Tracking a Locally Predictable Target among Obstacles, In Proc IEEE Int. Conf. on Robotics and Automation, 2006.

[8] V. Isler, S. Kannan, and S. Khanna, Randomized Pursuit-Evasion in a Polygonal Environment. IEEE Transactions on Robotics, 5(21):864$875,2005$.

[9] B. Jung and G. Sukhatme. Tracking targets using multiple robots: the effect of environment occlusion. In Journal Autonomous Robots, vol. 12 pp. 191-205, 2002.

[10] R. Murrieta-Cid and U. Ruiz and J.L. Marroquin and J.P. Laumond and S. Hutchinson, Tracking an Omnidirectional Evader with a Differential Drive Robot, Special Issue on Search and Pursuit/Evasion, Autonomous Robots, 31(4): 345-366, 2011.

[11] B. Tovar and S. M. LaValle: Visibility-based Pursuit - Evasion with Bounded Speed. I. J. Robotic Res, 27(11-12): 1350-1360, 2008.

[12] Merz A.W. The homicidal chauffeur a differential game. PhD. Thesis, Stanford University, 1971. 